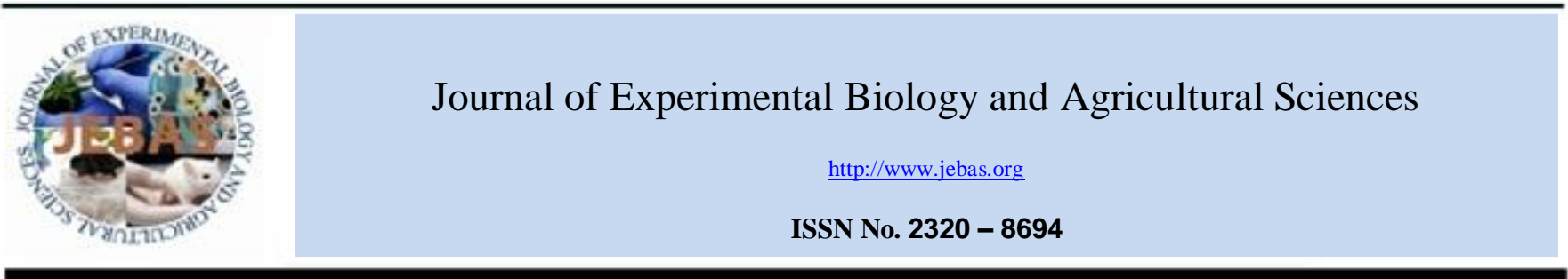

\title{
MAIZE MICRORNA ASSOCIATED ABIOTIC STRESSES: MINI REVIEW
}

\section{Sujatha E*, Saidulu Abbagoni}

Department of Botany, Osmania University, Hyderabad-500007, India.

Received - October 28, 2018; Revision - January 18, 2019; Accepted - February 25, 2019

Available Online - April 10, 2019

DOI: http://dx.doi.org/10.18006/2019.7(2).103.107

\section{KEYWORDS \\ miRNAs \\ Abiotic stress \\ Gene regulation \\ Drought \\ Salinity}

Radiation

\section{ABSTRACT}

Among the small RNA molecules, micro RNAs (miRNAs) are an interesting class of endogenous molecules that play a key role in the regulation of gene expression in various developmental and signaling cascades. Abiotic stress which induced abnormal expression in various miRNAs, can be a new target for developing stress tolerant breeds. Recent reviews show that during abiotic stress, miRNAs swamp the conditions by regulating target genes within the miRNA-target gene network and regulates various signaling pathways and crucial organ development like roots. Stress conditions leads to the stimulation and accumulation of positive regulators and suppression of negative regulators. The current review on miRNAs helps in identifying the role of miRNAs in stress management of maize crop. Study also help in establishing deviations in genetic profile of miRNAs during abiotic stress conditions. After compiling the available literature, it could be conclude that detail and deep studies are needed to understand the miRNA mediated regulatory mechanism to produce agriculturally important plants to endeavor the future needs.
* Corresponding author

E-mail: saidulugoud @gmail.com (Saidulu Abbagoni)

Peer review under responsibility of Journal of Experimental Biology and Agricultural Sciences.

Production and Hosting by Horizon Publisher India [HPI] (http://www.horizonpublisherindia.in/).

All rights reserved.
All the article published by Journal of Experimental Biology and Agricultural Sciences is licensed under a Creative Commons Attribution-NonCommercial 4.0 International License Based on a work at www.jebas.org.

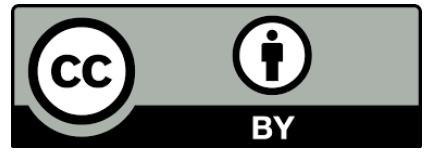




\section{Introduction}

Maize (Zea mays L.) is one of the widely cultivated primary crops for food and fodder throughout the world. Being an important cereal crop with diverse industrial applications, maize served as a model for the genetic and genomic research (Huang et al., 2002). Extensive research has been carried out on maize crop development and adaptation to the abiotic stress responses for continuing the better yield. Various environmental stress like salt, light, hypoxia, thermal, radiation, drought and oxidative stress severely affect the growth and development of the plant which subsequently decelerate the crop yield (Fahad et al., 2017). In recent years few attempts have been made for developing stress tolerant in this crop (Prasanna, 2016). It is also important to study the abiotic stress responses to understand the molecular changes in the genetic perspective. According to Hamilton \& Baulcombe (1999) RNA interference will be a better tool of choice for better understanding the gene regulation. Axtell \& Bowman (2008), reported that micro RNAs (miRNA) are the small RNAs which regulate the pre and post transcriptional modifications in protein synthesis during abiotic stress.

Plants have adopted various mechanisms to confront the unfavorable environmental stresses by regulating their gene expressions (Shukla et al., 2008). Insilico analysis of microRNAs and their target gene of stress induced Arabidopsis thaliana, and miRNA cloning had produced a new set of miRNAs that were not seen in the plants grown in stress-free conditions (Jones-Rhoades \& Bartel, 2004). By analyzing the complex miRNA-directed stress regulatory responses can provide new path for the development of stress tolerant plants (Wei et al., 2009). This present review outlines the association of miRNAs with abiotic stress.

\section{Biogenesis and Mechanism of Action of Plant Micro RNA}

Phillips et al. (2007) explained that plant micro RNAs are non coding RNAs, which consists of 20-24 nucleotides which encoded by miRNA genes. These genes can be transcribed into primary miRNAs by RNA polymerase II which can serve as miRNA precursors. This is a self-complementary and behaves like an imperfect double stranded structure. Dicer like (DCL) protein will produce the mature miRNA duplex from the precursor miRNA. Further, Chen (2005) reported that ARGONAUTE (AGO) protein degrades a single strand of the duplex which directs the AGO protein to recognize its perfect complementary nearby (target mRNA). This RNA associated with many actions like gene silencing which produces silencing complex (RISC), inhibits the protein synthesis by interfering in post translational modifications (Lanet et al., 2009).

\section{Role of MicroRNAs in Plant Abiotic Stress Responses}

Khraiwesh et al. (2012) suggested that every organism will try for survival; similarly, plants struggle to overcome the various biotic and abiotic stress conditions by gene regulations through different pathways. To combat these stress either they change the nature of gene regulation by over expression or suppression or sometimes by producing new miRNAs (Olejniczak et al., 2017). Sunkar \& Zhu (2004) reports suggested that Micro RNA profiling and microarray experiments of various models including A. thaliana explains the role of miRNAs in the stress conditions; some drought responsive miRNAs such as miR156, miR158, miR159, miR165, miR167, miR168, miR169, miR171, miR319, miR393, miR394 and miR397 were well identified in stress management (Sunkar et al., 2004). Salt shocked maize roots had downregulated miR156, miR164, miR167, and miR396 families and upregulated miR162, miR168, miR395 and miR474 families (Wei et al., 2009; Ding et al., 2009).

Micro RNAs can target multiple genes in a family but expression of specific target genes depends on the specific stress conditions (Sunkar et al., 2007; Sunkar et al., 2012). In case of maize plant, the miRNAs in high salinity shocks leads to the changes in transcription those are involved in various physiological and metabolic processes like organ formation, plant development and stress regulation (Jones-Rhoades et al., 2006). F-box auxin receptors like TIR1 (Transport Inhibitor Response Protein 1)/AFB (Auxin Signaling F-box Protein) were upregulated during drought stress in many plants such as Arabidopsis and rice (Chen et al., 2011). Deep gene sequencing of rice panicle in cold stress suppressed that the expression of miR1425 which targets genes of RNA binding proteins that encoding Pentatricopeptide repeat (PPR) proteins. Which have several functions like RNA stability, RNA splicing and RNA editing (Delannoy et al., 2007). Sunkar et al., (2006) reported that during oxidative stress gene transcripts CSD1, CSD2 and CSD3 that encodes for the Cu-Zn SODs were accumulated due to the down-regulation of miR398. Since, the superoxides cause oxidative damage to the plants which must be transformed into non toxic metabolites by ROS scavenging enzymes like superoxide dismutase (SOD).

\section{Identification and Target Prediction of Plant MiRNAs}

Due to the complex nature of miRNAs like methylation, multiple occurrences and small size, many sophisticated attempts in cloning methodologies have been adopted for the identification of miRNAs in plants, which resulted in various archives and databases for miRNAs (Tripathi et al., 2015). The miRBase contains 28,645 hair pin precursor miRNAs which express 35,828 mature miRNA products in 223 species including 73 plant species with 7057 miRNA loci (Kozomara \& Griffiths-Jones, 2014). 
miRNEST provides computational predictions and highthroughput sequencing results of miRNA data and now it contains more than 270 plant species and 22 viruse miRNAs including 2041 degradome data (Szczesniak et al., 2012). PMRD and PNRD represent plant miRNA sequences coupled with their targets. It consists of more than 8400 entries from around 120 plant species (Yi et al., 2015). The TAPIR uses two modes for predicting plant miRNAs, "Fast" mode and "Precise" mode (Bonnet et al., 2004). Evers et al. (2015) and Shiram et al., (2016) mentioned that there is a public access tool based on the functional similarity network for identification of plant-miRNA precursors, through application of transductive multi-label classification which is adaptable to heterogeneous and complex precursor populations.

\section{Abiotic Stress Induced Plant MiRNAs}

\subsection{MiRNAs in response to drought stress}

Aravind et al. (2017) explained about the structural and functional characterization of drought associated miRNAs in maize lead to the identification of 13 drought-associated miRNA families with 65 members which regulates 42 target mRNAs. The miR166 (Squamosa promoter-binding-like proteins) and miR395 (Growthregulating factors) families have expressed with various family specific targets as high as 14 miRNAs in each family. But, zmamiR160, zma-miR390, zma-miR393, and zma-miR2275 expressed a single target only. The target mRNAs has coupled with NAC, GAMYB and HD-Zip which are few drought-related transcription factors. In drought tolerant maize (HKI-1532) it is observed that $35 \%$ of miRNAs were up-regulated and in drought sensitive maize (V-372) 31\% of miRNAs were up-regulated (Aravind et al., 2017). Moreover, the target mRNAs was up regulated in HKI-1532 by $14.2 \%$ and in V-372 it is $2.38 \%$ only. Interestingly, the expression patterns of miRNA-target mRNA pairs for HKI-1532 showed 46 Type I, 13 Type II, and 23 Type III patterns, but, V-372 displayed Type IV interactions (151). In the stress tolerant maize, the fundamental and essential biological functions like endosperm expression, auxin \& light responsive pathway and ABA signaling were maintained by less accumulation and down regulation of few miRNAs.

\subsection{MiRNAs in response to salinity stress}

Role of miRNAs in management of salinity stress was also recognized by Yang et al. (2014). In addition to the synthesis of proteins, translation IFs plays various other important roles in plants. Translational initiation factors like IF1 can help the plant to tolerate the abiotic stress responses through various mechanisms. Tamarix hispida roots and stems have expressed IF1A after 6 and 48 hours of salinity shock respectively. This salinity resistant nature was adopted by regulating the biosynthesis of proline which is an osmolytic in salinity stress.
This accumulation is caused by the up regulation of Pyrroline-5carboxylate synthetase (P5CS isoform 1) (GRMZM2G375504) related translation (Strizov et al., 1997). In salt stress few miRNAs, such as mir_250 vs. glutathione peroxidase (GPX) and DNA (cytosine- 5-)-methyltransferase, mir_316 vs. gibberellin receptor (GID1), mir_330 vs. casein kinase II subunit alpha (CK2a) and cysteine proteinase inhibitor, and mir_316 vs. sucrose-phosphatase-1 (SPP1) in leaves, roots, or both were transregulated (Fu et al., 2017). Proline dehydrogenase targeted genes like miR474 have been identified to be up regulated in abiotic stress like drought in maize (Wei et al., 2009).

\section{3 miRNAs in response to hypoxia}

Hypoxia or anaerobic conditions altered the expressions of 39 miRNAs in maize. Auxin response factor, SCL, HD-ZIP and the WRKY domain proteins were accumulated because of the induction of various miRNAs (zma-miRNA166, zma-miRNA167, zma-miRNA171 and osa-miRNA396) at four different submergence time points in the early submergence phases.

Moreover, few miRNAs (zma-miR159, ath-miR395-like, ptcmiR474-like and osa-miR528) have been reduced at early submergence phase but induced after one day of submergence. All the miRNA targets involve in carbohydrate and energy metabolism and they possess the cis-acting element, which is necessary for the hypoxia or hormone induction (Zhang et al., 2008).

\subsection{MiRNAs in response to radiation}

Shen et al. (2015) reported that UV-B radiation in the seedlings of maize leads to the increased levels of anthocyanin and phenol. UV-B radiation causes severe membrane damage leads to lipid peroxidation and osmolytic leakage. Among the 17 UV-Bresponsive miRNAs in irradiated maize, seven miRNAs (miR164, miR165, miR166, and miR398) were up regulated by UV-B, and 10 miRNAs (miR164, miR165, miR166, and miR398) were down regulated. Some miRNAs like miR156, miR171, and miR172 can switch various growth stages of the plant life cycle and some miRNAs like miR156, miR171, and miR172 will regulate these transitions (Casati, 2013).

\section{Conclusion}

From the current literature, it can be perceived that the numerous efforts of the scientists exemplified the mechanisms associated with plant abiotic stress response. To swamp the future problems, it is necessary to produce new breeds and stress tolerant crops. The continuous research on the identification of small RNAs and protein-coding genes for abiotic stress, few researches are available about the exact roles of these miRNAs. Though much advancement like computer aided sequencing technologies and 
databases are available, this area is still in infant stage. The aberrant expressions of miRNAs, especially which are associated in abiotic stress are extremely important in order to develop drought tolerant and genetically improved plants. It is obvious from the past that there is a need to explore the new targets to develop agriculturally important crop varieties. Development of artificial miRNAs is also an important approach for designing stress tolerated crops. It is also important to investigate the reason for stress response. The response may be due to genotypic changes or abiotic stress. This can be affirmed by large scale models using different plant species. It is observed that miRNAs can be modified in the cascade of biosynthesis. It is very important to identify whether the modifications like truncations, nucleoside substitution and addition are associated with the abiotic stress or not for better understanding of the stress response.

\section{Conflict of Interest: Nil}

\section{References}

Aravind J, Rinku S, Pooja B, Shikha M, Kaliyugam S, Mallikarjuna MG, Kumar A, Rao AR, Nepolean T (2017) Identification, Characterization, and Functional Validation of Drought-responsive MicroRNAs in Subtropical Maize In breds. Frontiers in Plant Science 8: 941.

Axtell MJ, Bowman JL (2008) Evolution of plant microRNAs and their targets. Trends Plant Science 13: 343-349.

Bonnet E, Wuyts J, Rouze P, Vande Peer (2004) Detection of 91 potential conserved plant microRNAs in Arabidopsis thaliana and Oryza sativa identifies important target genes. Proceedings of the National Academy of Sciences 101: 11511-11516.

Casati P (2013) Analysis of UV-B regulated miRNAs and their targets in maize leaves. Plant Signaling and Behavior 8: 26758.

Chen X (2005) MicroRNA biogenesis and function in plants. FEBS Letters 579: 5923-5931.

Chen ZH, Bao ML, Sun YZ, Yang YJ, Xu XH (2011) Regulation of auxin response by miR393-targeted transport inhibitor response protein 1 is involved in normal development in Arabidopsis. Plant Molecular Biology 77: 619-629.

Delannoy E, Stanley WA, Bond CS, Small ID (2007) Pentatricopeptide repeat (PPR) proteins as sequence-specificity factors in posttranscriptional processes in organelles. Biochemical Society Transactions 35: 1643-1647.

Ding D, Zhang L, Wang H, Liu Z, Zhang Z (2009) Differential expression of miRNAs in response to salt stress in maize roots. Annals of Botany 103: 29-38.
Evers M, Huttner M, Dueck A, Meister G, Engelmann JC (2015) miRNA, adaptable novel miRNA identification in plants using small RNA sequencing data. BMC Bioinformatics 16: 370 .

Fahad S, Bajwa AA, Nazir U, Anjum SA, Farooq A, Zohaib A, Sadia S, Nasim W, Adkins S, Saud S, Ihsan MZ, Alharby A, Wu C, Wang D, Huang J (2017) Crop Production under Drought and Heat Stress: Plant Responses and Management Options. Frontiers in Plant Science 8:1147.

Fu R, Zhang M, Zhao Y, He X, Ding C, Wang S, Feng Y, Song X, Li P, Wang B (2017) identification of salt tolerance-related microRNAs and their targets in Maize (Zea mays L.) using highthroughput sequencing and degradome analysis. Frontiers in Plant Science 8: 864 .

Hamilton AJ, Baulcombe DC (1999) A species of small antisense RNA in post transcriptional gene silencing in plants. Science 286 : 950-952.

Huang J, Pray C, Rozelle S (2002) Enhancing the crops to feed the poor. Nature 418: 678-684.

Jones Rhoades MW, Bartel DP, Bartel B (2006) MicroRNAs and their regulatory roles in plants. Annual Review of Plant Biology 57: 19-53.

Jones Rhoades MW, Bartel DP (2004) Computational identification of plant micro- RNAs and their targets, including a stress-induced miRNA. Molecular Cell 14: 787-799.

Khraiwesh B, Zhu JK, Zhu J (2012) Role of miRNAs and siRNAs in biotic and abiotic stress responses of plants. Biochim Biophysica Acta 1819: 137-148.

Kozomara A, Griffiths-Jones S (2014) miRBase, annotating high confidence micro RNAs using deep sequencing data. Nucleic Acids Research 42: 68-73.

Lanet E, Delannoy E, Sormani R, Floris M, Brodersen P (2009) Biochemical evidence for translational repression by Arabidopsis microRNAs. Plant Cell 21: 1762-1768.

Olejniczak M, Kotowska-Zimmer A, Krzyzosiak W (2017) Stress-induced changes in miRNA biogenesis and functioning. Cellular and Molecular Life Sciences 75 :177-191.

Phillips JR, Dalmay T, Bartels D (2007) The role of small RNAs in abiotic stress. FEBS Letter 581: 3592-3597.

Prasanna BM (2016) Developing and deploying abiotic stresstolerant maize varieties in the tropics: challenges and opportunities. molecular breeding for sustainable crop improvement. Sustainable Development and Biodiversity 11: 61-77 
Shen X, Dong Z, Chen Y (2015) Drought and UV-B radiation effect on photosynthesis and antioxidant parameters in soybean and maize. Acta Physiologiae Plantarum 37: 25.

Shriram V, Kumar V, Devarumath RM, Khare TS, Wani SH (2016) MicroRNAs as potential targets for abiotic stress tolerance in plants. Frontiers in Plant Science 7: 817.

Shukla LI, Chinnusamy V, Sunkar R (2008) The role of microRNAs and other endogenous small RNAs in plant stress responses. Biochimica et Biophysica Acta 1779: 743-748.

Strizhov N, Ábrahám E, Ökrész L, Blickling S, Zilberstein A, Schell J (1997) Differential expression of two P5CS genes controlling proline accumulation during salt-stress requires $\mathrm{ABA}$ and is regulated by ABA1, ABI1 and AXR2 in Arabidopsis. The Plant Journal 12: 557-569.

Sunkar R, Chinnusamy V, Zhu JH, Zhu JK (2007) Small RNAs as big players in plant abiotic stress responses and nutrient deprivation. Trends Plant Science 12: 301-309.

Sunkar R, Kapoor A, Zhu JK (2006) Post transcriptional induction of two $\mathrm{Cu} / \mathrm{Zn}$ superoxide dismutase genes in Arabidopsis is mediated by down regulation of miR398 and important for oxidative stress tolerance. Plant Cell 18: 2051-2065.

Sunkar R, Li YF, Jagadeeswaran G (2012) Functions of microRNAs in plant stress responses. Trends in Plant Science 17: 196-203.
Sunkar R, Zhu JK (2004) Novel and stress-regulated microRNAs and other small RNAs from Arabidopsis. Plant Cell 16: 2001-2019.

Szczesniak MW, Deorowicz S, Gapski J, Kaczyñski \& Makałowska I (2012) miRNEST database, an integrative approach in micro RNA search and annotation. Nucleic Acids Research 40: 198-204.

Tripathi A, Goswami K, Sanan-Mishra N (2015) Role of bioinformatics in establishing micro RNAs as modulators of abiotic stress responses, the new revolution. Frontiers in Physiology 6: 286.

Wei L, Zhang D, Xiang F, Zhang Z (2009) Differentially expressed miRNAs potentially involved in the regulation of defence mechanism to drought stress in maize seedlings. International Journal of Plant Sciences 170: 979-989.

Yang G, Yu L, Zhao Y, Zhao Z Gao C (2014) Stress tolerance analysis of a Tamarix hispida -The IF1A in Saccharomyces cerevisiae. Journal of Nanjing Forestry University 38: 62-66.

Yi X, Zhang Z, Ling Y, Xu W, Su Z (2015) PNRD, a plant noncoding RNA database. Nucleic Acids Research 43: 982-989.

Zhang Z, Wei L, Zou X, Tao Y, Liu Z, Zheng Y (2008) Submergence responsive MicroRNAs are potentially involved in the regulation of morphological and metabolic adaptations in maize root cells. Annals of Botany 102: 509-519. 